\title{
Activity on a Li-rich giant: DI Psc revisited
}

\author{
Levente Kriskovics, Zsolt Kővári, Krisztián Vida and Katalin Oláh \\ Konkoly Observatory, \\ Konkoly Thege út 15-17., H-1121, Budapest, Hungary \\ email: kriskovics, kovari, vidakris, olah@konkoly.hu
}

\begin{abstract}
We present a new Doppler imaging study for the Li-rich single K-giant DI Psc. Surface temperature maps are reconstructed for two subsequent rotation cycles. From the time evolution of the spot distribution antisolar-type differential rotation pattern is revealed. We show marks of non-uniform Li-abundance as well. The possible connection between the current evolutionary phase of the star and its magnetic activity is briefly discussed.
\end{abstract}

Keywords. stars: activity, stars: imaging, stars: individual (DI Psc), stars: spots, stars: late-type

\section{Introduction}

DIPsc (HD 217352) is a rapidly rotating ( $P_{\text {rot }}=18.07$ days $)$ single K-giant, a new candidate for the small group of Li-rich K-giant stars. The extreme Li-abundance is related to a short evolutionary episode, the helium flash, when different (partly unknown) processes activate Li-production and propagation (Charbonnel \& Balachandran 2000). Moreover, the role of rotation and magnetic activity in these processes is also unclear. Surface Li-abundance and the position of DI Psc on the HRD was determined in our recent Doppler imaging study (Kővári et al. 2013). In this paper we aim to investigate the time evolution of the surface by Doppler imaging, as well as the Li-distribution on the surface.

\section{Doppler imaging results}

15 time series spectra of exceptionally high signal-to-noise ratio were taken with NARVAL@TBL in Nov-Dec 2012, covering 40 days (i.e., $\approx 2 \times P_{\text {rot }}$ ). We use the Doppler imaging code TempMap by Rice et al. (1989) for three lines (Fe I-6430, Ca I-6439 and Li I$6708)$ to reconstruct the stellar surface in two consecutive rotational cycles. Combined $(\mathrm{Fe}+\mathrm{Ca}+\mathrm{Li})$ images, as well as their cross-correlation is plotted in Fig. 1. The best fit correlation pattern suggests antisolar-type surface differential rotation with a shear of $\alpha=-0.11 \pm 0.02$. This result should be regarded as a preliminary one, since unexpected rearrangements in spot configuration (e.g., emerging new flux) can disturb seriously the
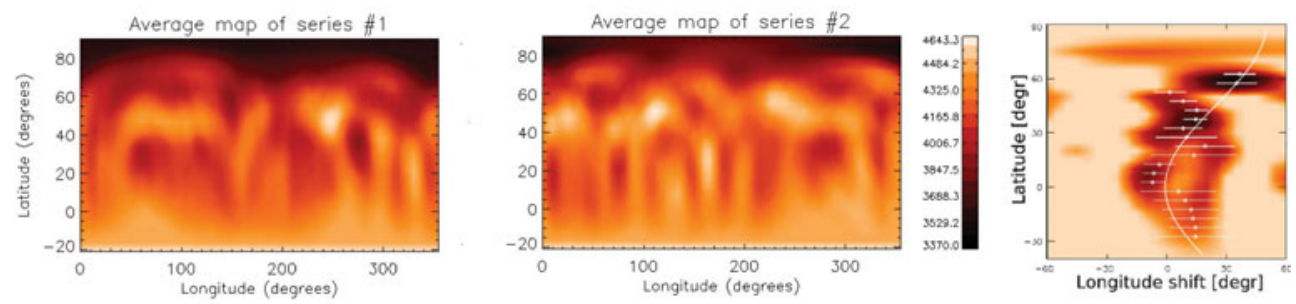

Figure 1. Combined Doppler images for two consecutive rotation periods (left) in Nov-Dec 2012 and the fitted antisolar-type differential rotation on the cross-correlation function map (right). 

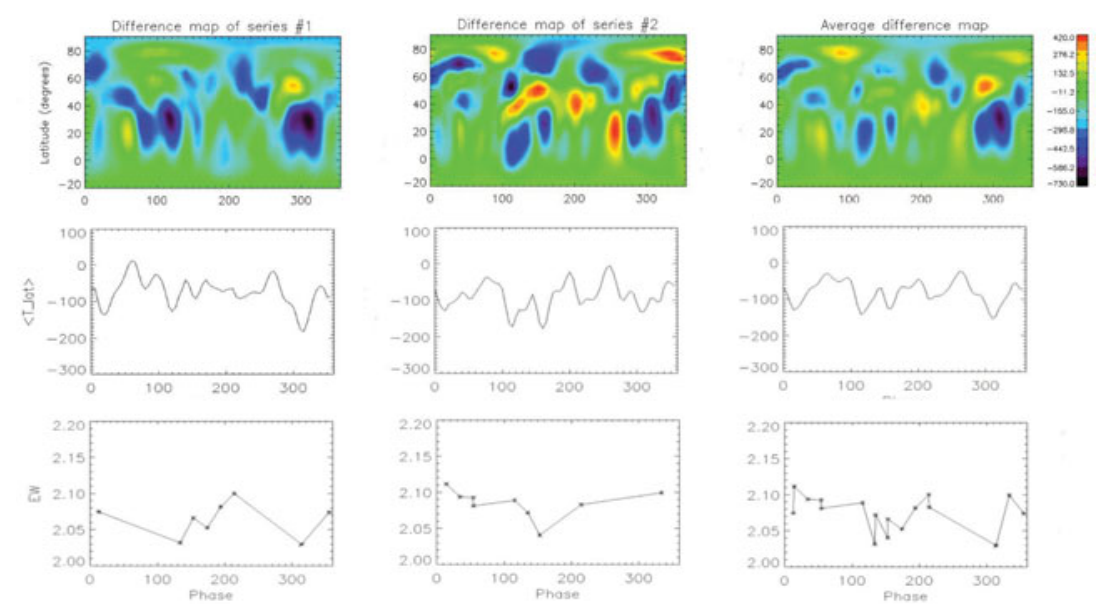

Figure 2. Top: difference maps (i.e., Li-maps are subtracted from the average maps shown in Fig 1 left). Note the similar structure for both rotational cycles. Middle: longitudinal distribution of the mean latitudinal temperature. Bottom: rotational modulation of the Li I-6708 equivalent width.

pure differential rotation pattern, thus yielding false observation. We note, however, that antisolar-type differential rotation was reported also for DP CVn, which is another Li-rich K-giant, the twin of DI Psc (Kövári et al. 2013). High Li-abundance might be related to strong meridional circulation (i.e., extra mixing), which, on the other hand, can eventuate antisolar-type differential rotation (cf. Kitchatinov \& Rüdiger 2004).

Non-uniform surface Li-abundance would affect the Doppler maps by altering the strength of the local line profiles (the temperature inversion code assumes constant abundance, therefore a higher $\mathrm{Li}$ equivalent width is fitted with a lower temperature causing a false cool spot on the map). In order to investigate this behaviour we subtract the Limaps from the combined $(\mathrm{Fe}+\mathrm{Ca}+\mathrm{Li})$ ones. The resulting difference maps are plotted in Fig. 2 top, where signs of similar non-uniformity can be marked in both rotation cycles. This is supported by the longitudinal distribution of the mean latitudinal temperature values, as well as by the rotational modulation of the Li I-6708 equivalent width (middle and bottom panels of Fig. 2, respectively). We conclude that DI Psc is an optimal target for studying the connection between surface activity, rotation and surface Li-enrichment.

\section{Acknowledgements}

This work has been supported by the Hungarian Science Research Program OTKA K-81421, the Lendület-2009 and Lendület-2012 Young Researchers' Programs of the Hungarian Academy of Sciences and by the HUMAN MB08C 81013 grant of the MAG Zrt.

\section{References}

Charbonnel, C. \& Balachadran, S. 2000, A\&A, 309, 563

Kitchatinov, L. L. \& Rüdiger, G. 2004, AN, 325., 496

Kővári, Zs., Korhonen, H., Strassmeier, K. G., et al. 2013, A\&ऽA, 551., 2

Rice, J. B., Wehlau, W. H., \& Khokhlova, V. L. 1989, A\& A, 208, 179 